\title{
The Mysterious Case of Maternal Heartbeat Sounds
}

Philbin MK

Independent Researcher, Moorestown, NJ, USA

\section{Aims and Methods}

Many people believe that maternal heartbeat sounds dominate the uterine environment and that the fetus, preterm and term newborn prefer them. ${ }^{1,2,3}$ This presentation critically examines the literature addressing this belief.

\section{Results}

In 1962, Lee Salk, a psychiatrist in New York City, took a walk through the zoo and noticed a monkey holding her infant close to her body in her left arm "closest to her heart". ${ }^{1,2}$ In 40 out of 42 subsequent observations, this one monkey did the same. With these and data from observations of newly delivered women and their infants, Salk concluded that every primate is imprinted to their mother's heartbeat during infancy because each female holds her own infant on the left to experience "the pleasurable sensation of her own heartbeat reflected back from the infant". Thus, behavior due to each mother's own imprinting passes it to the next generation. ${ }^{2,4,5}$ Extrapolating lavishly, Salk proposed heartbeat sounds as "the basis of all later learning" and that a "universal, ...biological tendency to seek heartbeat sounds has survival value [and] ....involves mutual satisfaction." ${ }^{2}$ Salk's work was influential in bringing the importance of maternalinfant closeness to professional attention. ${ }^{6}$

With numerous, unwitting errors Salk tested the theory of lifetime heartbeat imprinting in a foundling (orphan) hospital ${ }^{2}$ by comparing tape recorded nighttime sounds emitted in whole rooms of healthy infants or toddlers. One room had broadcast heartbeat sounds and the other had "no sounds" (actually room sounds) or broadcast lullabies. The conditions were not masked, and baby nurse activities were not reported. Because the number of infants making sounds was not determined, even one infant could account for all room sounds. The heartbeat condition always had fewer sounds (more sleep) than the control conditions.

In 1968 and 1970 several obstetricians sought to extend heartbeat imprinting into fetal life by recording sounds in utero from unconscious women in labor. ${ }^{7,8}$ Although the results were determined by methodological errors, these are the studies that catapulted intrauterine heartbeat sounds into the popular culture where they remain stuck. The emotionally attractive idea of influential intrauterine sounds accounts, in part, for the dangerous practice of propagating all kinds of sounds in the uterus via speakers attached to the pregnant belly or inserted in the vagina.

A responding study using appropriate methods and equipment did not find heartbeat sounds in the uterus of conscious laboring women with a spinal block but did find room and maternal voice sounds. ${ }^{9}$

Studies of heartbeat recognition in infancy generally show preferential responding to them. However, the findings may be due to too great a difference between experimental and control sounds ${ }^{10,11,12,13}$ heartbeat sounds may be preferable only because they are simple. But a newborn's ability to make fine discriminations ${ }^{14,15,16,17,18}$ enables contrast stimuli differing only in rhythm. There is no clear preference indicating that newborns have not had exposure to heartbeats. (Such a study has not been found.)

Giving up a long-held belief is difficult even when alternatives are substantial. ${ }^{19}$ Thankfully, the alternative to intrauterine heartbeats is gold, namely mother's voice. It, and not prominent heartbeats, has been found reliably in the pregnant uterus of humans and ewes. ${ }^{9} 20$ Well-known investigators conclude, "mother's voice... [is] the most significant and common mode of potential acoustic stimulation in the uterus." 20

\section{Conclusions}

Credible research shows that heartbeat sounds are not distinguishable in utero but that discriminable features of mother's voice are prominent - a necessary condition to eventual language acquisition. A broad, moral-of-the-story conclusion is that there is nothing quite like a tour through primary sources to examine a common belief.

References

1. Salk L. The effects of the normal heartbeat sound on the behavior of the newborn infant; implications for mental health. World Mental Health 1960,12:168-175.

2. Salk L. Mother's heartbeat as an imprinting stimulus. Transactions of the New York Academy of Sciences 1962 April 10, 1962:753- 763.

3. Panagiotidis J, Lahav A. Simulation of prenatal maternal sounds in NICU incubators: a pilot safety and feasibility study. The Journal of Maternal-Fetal and Neonatal Medicine 2010, 23:106-109.

4. Hess EH. Imprinting. Science. 1959,130:133-141.

5. Moltz H. Imprinting: empirical basis and theoretical significance. Psychological Bulletin 1960,57:291-314.

6. Salk L. The role of heartbeat in the relations between mother and infant. Scientific American 1973,228:24-29.

7. Bench JR. Sound transmission to the human foetus through the maternal abdominal wall. Journal of Genetic Psychology 1968,113: 85-87.

8. Grimwade JC, Walker DW, Wood C. Sensory stimulation of the human fetus. Australian Journal of Mental Retardation 1970,2:63-64.

9. Richards DS, Frentzen B, Gerhardt KJ, McCann ME, Abrams RA. Sound levels in the human uterus. Obstetrics and Gynecology 1992,89:186 - 190.

10. Panagiotidis J, Lahav A. Simulation of prenatal maternal sounds in NICU incubators: a pilot safety and feasibility study. The Journal of Maternal-Fetal and Neonatal Medicine 2010,23(S3):106-109

11. Ullal-Gupta S, Vanden Bosch der Nederlanden CM, Tichko P, Lahav A, and Hannon E. Linking prenatal experience to the emerging musical Mind. Frontiers in. Systematic Neuroscience 2013,7:48.

12. Rand K, Lahav A. Impact of the NICU environment on language deprivation in preterm infants. Acta Pediatrica 2014,103:245-248.

13. Doheny L, Hurwitz S, Insoft R, Ringer S, Lahav A. Exposure to biological maternal sounds improves cardiorespiratory regulation in extremely premature infants. The Journal of Fetal and Neonatal Medicine. 2012;25:1591-1594.

14. Shahidullah S, Hepper PG. Frequency discrimination by the fetus. Early Human Development. $1994 ; 36: 13-26$. 
15. Moon C, Lagercrantz H, Kuhn PK. Language experienced in utero affects vowel perception after birth: a two-country study. Acta Pediatrica. 2013;102:156-60.

16. DeCasper AJ, Fifer WP. Of human bonding: newborns prefer their mothers' voices. Science, New Series. 1980;208:1174-1176.

17. DeCasper AJ, Prescott. Lateralized processes constrain auditory reinforcement in human newborns. Hearing Research. 1984;255.135-141.

18. Spence M, DeCasper A. Prenatal experience with low-frequency maternal-voice sounds influence perception of maternal voice samples. Infant Behavior and Development. $1987 ; 16,133-142$.
19. Kuhn TS. The Structure of Scientific Revolutions: 50th Anniversary Edition. 4th Ed. Chicago, IL: University of Chicago Press; 2012. pp.264.

20. Abrams RM, Gerhardt KJ. (2000) The acoustic environment and physiological responses of the fetus. Journal of Perinatology. 2000;20(part 2): S31 - S36.
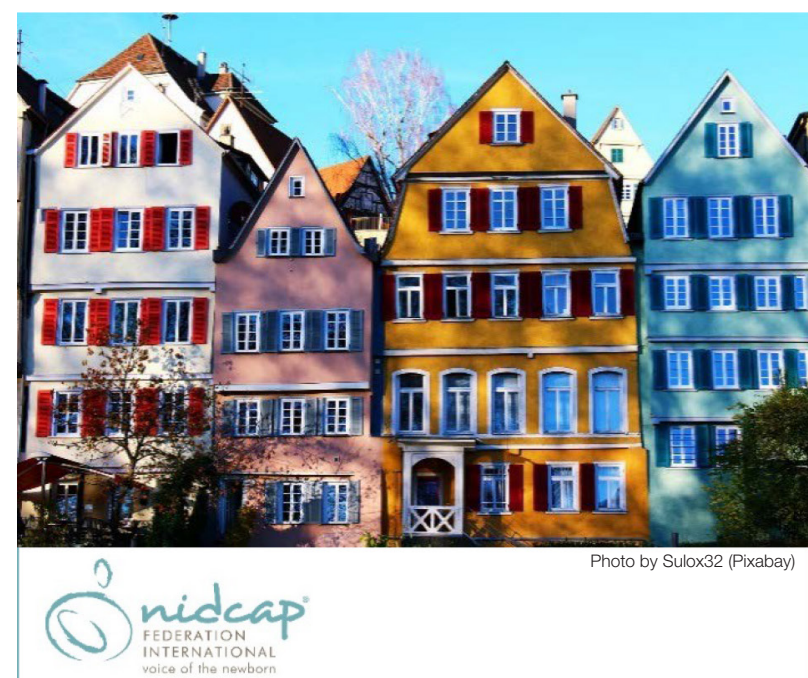

$31^{\text {st }}$ Annual

\section{NIDCAP Trainers Meeting}

The Impact of NIDCAP on

Infant, Family, and Society

October 24-26, 2020

Bernhäuser Forst Conference Center Filderstadt, Germany

www.berhaeuser-forst.de

Hosted by NIDCAP Germany, NIDCAP Training Center Tübingen on behalf of the NFI

(By Invitation Only)

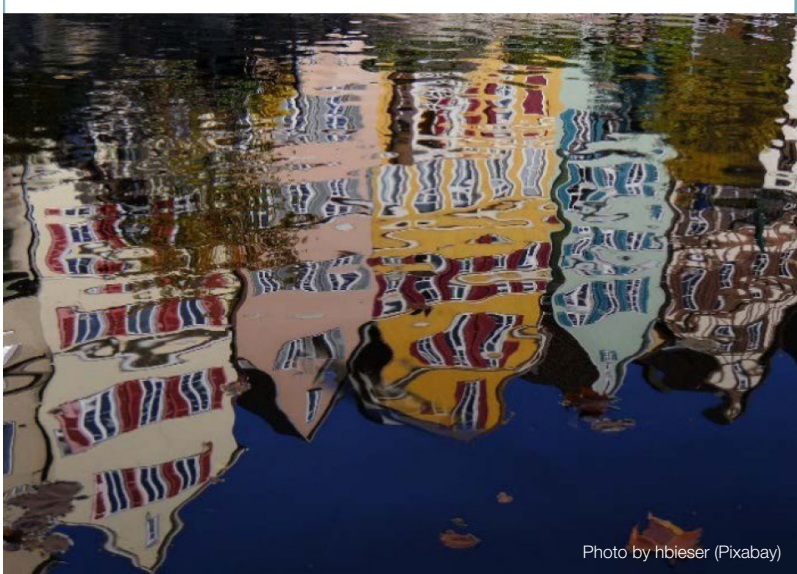

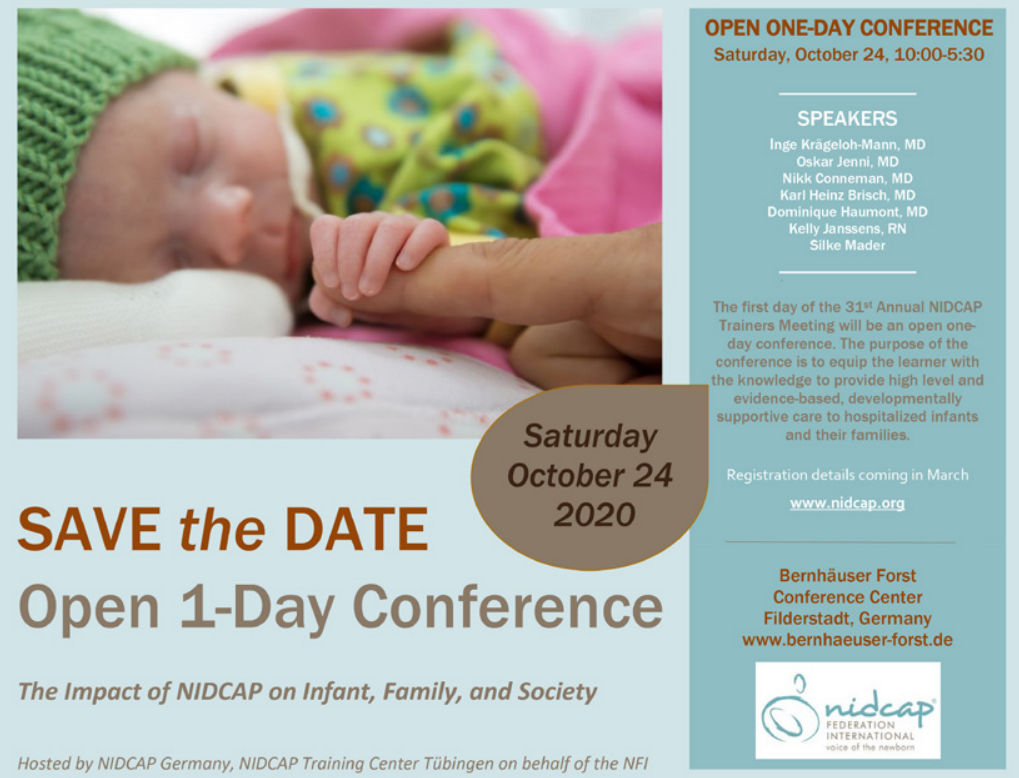

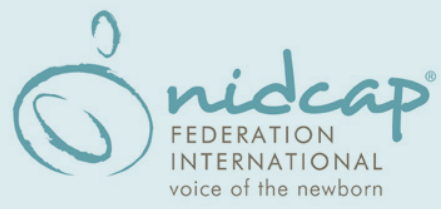

Mission

The NFI promotes the advancement of the philosophy and science of NIDCAP care and assures the quality of NIDCAP education, training, mentoring and certification for professionals, and hospital systems.

Adopted by the NFI Board, July 1, 2019

\section{Vision}

The NFI envisions a global society in which all hospitalized newborns and their families receive care in the evidencebased NIDCAP model. NIDCAP supports development, enhances strengths and minimizes stress for infants, family and staff who care for them. It is individualized and uses a relationship-based, family-integrated approach that yields measurable outcomes.

Adopted by the NFI Board, October 20, 2017 